\title{
Biosynthesis and Biotransformations of Biopolymers: Starch and Poly(hydroxyalkanoates)
}

\author{
Viviana Garcés ${ }^{1,2}$, Manuel Palencia 2 \\ ${ }^{1}$ Mindtech Research Group (Mindtech-RG), Mindtech s.a.s., Cali-Colombia \\ ${ }^{2}$ GI-CAT, Department of Chemistry, Universidad del Valle, Cali-Colombia
}

Corresponding Author: M. Palencia. E-mail: manuel.palencia@correounivalle.edu.co
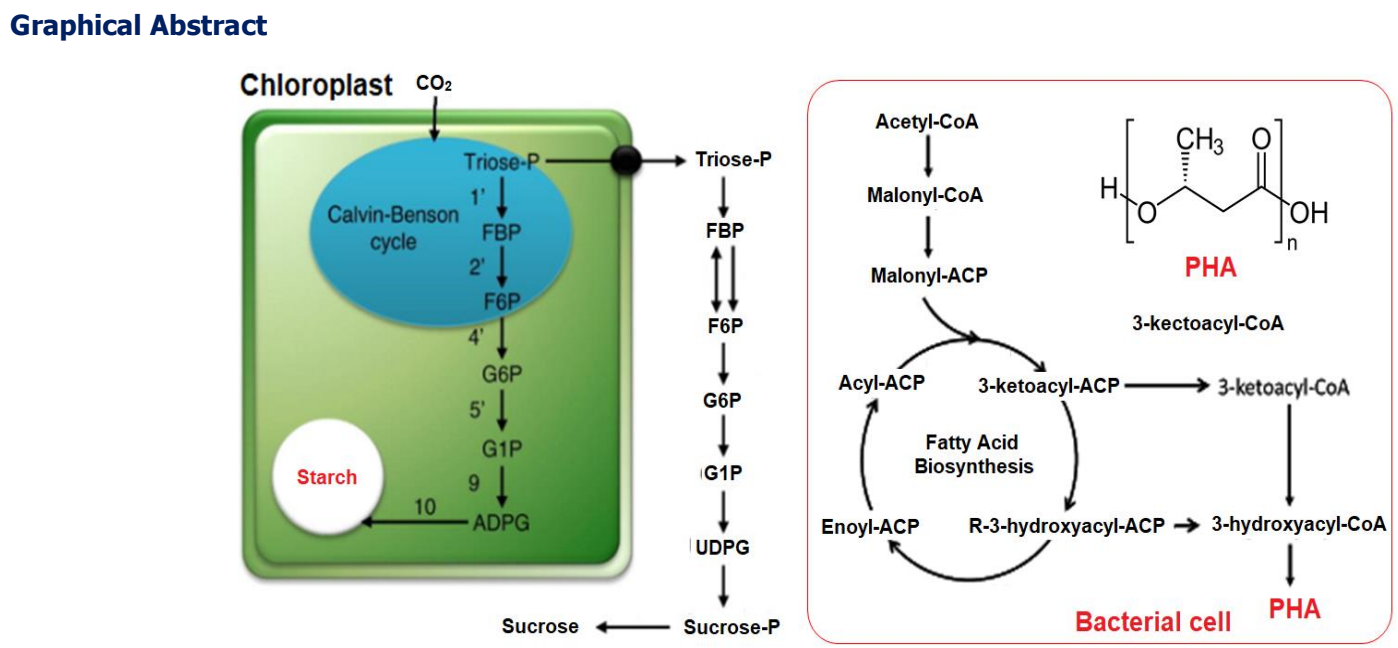

Abstract. Processes mediated by living organisms, animal, plants, fungi, algae, bacterial and virus have been the center of attention for many investigations looking for new sustainable alternatives for the development of new material. Biosynthetic pathways have emerged as a strategy to advance in the development of eco-friendly industry since they are the result of evolutionary and adaptive processes which have perfected the enzymatic machinery behind biotransformations and other biosynthetic processes. This review shows a general view about the biosynthetic processes for the making of biopolymers of interest for the industry, but at the same time, biotransformations for the obtaining of precursor, polymers and raw material are included. In particular, it is focused on starch and poly(hydroxyalkanoates) due to its industrial use and potential for the making of bioplastics.

Keywords: Biosynthesis, starch, poly(hydroxyalkanoate), bacterial cell, chloroplasts, biopolymer, biotransformation, fermentation

Cite as: Garcés V., Palencia M. Biosynthesis and Biotransformations of Biopolymers: Starch and Poly(hydroxyalkanoates) - A Review. J. Sci. Technol. Appl. 9 (2020) 4-9.

\section{Accepted: 2020-11-10 \\ Published: 2020-11-15 \\ Paper Number: 060} (c) (1) (3) (2)
This is an open access article distributed under the terms of the Creative Commons Atributtion License

\section{Minireview}

(c) MT-Pallantia Publisher 2020 


\section{Content}

1. Introduction: Biosynthesis and polymer biotransformations

2. Starch biosynthesis

3. Biosynthesis of poly(hydroxyalkanoates)

4. Biotransformation of polymers

5. Conclusions

8. References

\section{Introduction}

Currently, environmental concerns and the decrease in fossil fuel reserves have generated great interest in green and sustainable chemistry, with the research and development of bio-based materials or products. As explained in the previous chapter, the raw material used to obtain biopolymers has its origin in living organisms, therefore, a question that from this statement is: How are biopolymers produced?

To talk about the way how biopolymers are produced, one would have to introduce a concept that we assume we understand, but it is better to remember it and it is the concept of biosynthesis. According to IUPAC, biosynthesis is defined as "the production of a chemical compound by a living organism" (IUPAC, 1997). In other words, in living organisms such as humans, plants, fungi, algae, and bacteria, biosynthesis is the metabolic process in which biomolecules or complex molecules with specific functions are synthesized from chemical reactions catalyzed by enzymes from small molecules.

This minireview will discuss the biosynthesis processes for starch and poly(hydroxyalkanoates) (PHAs) due to the industrial importance of them for applications as bioplastics. In the first case, we will talk about starch that is biosynthesized by plants and stored in leaves, stems and roots, and secondly, the PHAs biosynthesized by bacteria, archaea and algae. To more detailed review and conceptualization into environmental, sustainability, synthetic and application aspects related with biopolymers, bio-based polymers and eco-friendly polymers, it is recommended to consult the book "Eco-friendly Functional Polymers: An Approach from Application-Targeted Green Chemistry" (Elsevier, Palencia et al.).

\section{Starch biosynthesis}

Starch is the most abundant source of energy in plants, it is responsible for most cellular processes, and it's located in their leaves (chloroplasts), stem, roots, seeds and fruits. Commercially, the starch is extracted from grains such as corn, rice, barley and oats, from banana, potato, sweet potato, yam, sweet potato and yucca. In addition, let us remember that starch is biopolymers made up of two polymeric units of glucose, amylose and amylopectin (Karan et al., 2020; Verma et al., 2020; Kosseva et al., 2020).

During the photosynthesis process (which is the process by which certain plants, bacteria and algae, transform solar energy into chemical energy by producing monosaccharides that, through cellular respiration, convert into adenosine triphosphate (ATP), this process is associated with the green pigment called chlorophyll)
Much of the carbon is stored in the chloroplasts located in the leaves for the synthesis of starch, and during the night it is mobilized to promote plant growth, starch storage and other metabolic processes (Verma et al., 2020; Clasen et al., 2018 ; Tetlow et al., 2011). Unlike the starch that is synthesized during the day in the chloroplasts and that is used in multiple subsequent processes, the long-term storage of the starch is located in the amyloplasts; which are a type of plastids found in high quantities in non-photosynthetic reserve tissues, such as endosperm in seeds and tubers (Orzechowski, 2008). This leads to structural differences between the starch present in amyloplasts with respect to that found transiently in chloroplasts and scientifically it has been affirmed that the starch present in the latter has a lower content of amylose. This difference between the metabolic pathways of chloroplasts and amyloplasts has led to reflection on the true processes carried out in these two organelles, accepting that there are differences in the synthetic pathways in photosynthetic and non-photosynthetic cells (Li et al., 2019; Orzechowski, 2008).

In the case of chloroplasts, it is suggested that starch synthesis is the end product of the metabolic pathway that is linked to the CalvinBenson cycle. The Calvin-Benson cycle can be very long, but it can be summarized in three steps; first the union of two triose phosphate gives rise to a fructose-1,6-bisphosphate (Triose-P), then the enzyme fructose-1,6-bisphosphatase (FBP) converts fructose-1,6bisphosphate to fructose-6-phosphate (F6P). The F6P resulting from this cycle is isomerized by pPGI (plastid phosphoglucose isomerase) to glucose-6-phosphate (G6P). In the next step, plastid phosphoglucomutase (pPGM) converts G6P into glucose-1phosphate (G1P), at this point in the metabolic pathway ADPG pyrophosphorylase (AGP) is used and an ATP molecule is inverted and to obtain ADP-glucose (ADPG) and inorganic pyrophosphate (PPi) which is hydrolyzed to inorganic phosphate by the action of a pyrophosphorylase. Finally, ADPG is the unit that is added to amylose and amylopectin chains by different additional metabolic pathways (Bahaji et al., 2014; Miao et al., 2014; Varela et al., 2008). For amylose which is a linear polymer linked by $\alpha-1,4$-glucosidic bonds, chain elongation is serialized through granule-bound starch synthase (GBSS) when an ADPG residue is transferred to glucan substrates. to produce relatively long chain amylose molecules. On the other hand, in the case of amylopectin which is a branched polymer linked by $\alpha-1,4$-glucosidic and $\alpha-1,6$-glucosidic bonds, its synthesis requires soluble starch synthases (SS), starch branching enzymes (SBE) and starch debranching enzymes (DBE), see Figure 1, (Miao et al., 2014).

Starch exhibits the characteristic of hydrophilicity, which helps its biodegradability, but deteriorates its mechanical properties. Starch modification is a methodology used in several areas to change physicochemical characteristics. There are several techniques for starch modification, including: (i) chemical modification, (ii) physical modification, (iii) enzymatic modification, and iv) genetic modification (Wang et al., 2020). These modifications are made in search of hydrophobic characteristics, which allows the use of starch as emulsions, pharmaceutical excipients, packaging materials, in polymer mixtures and others (Wang et al., 2020; Chen et al., 2018; Haroon et al., 2016). 


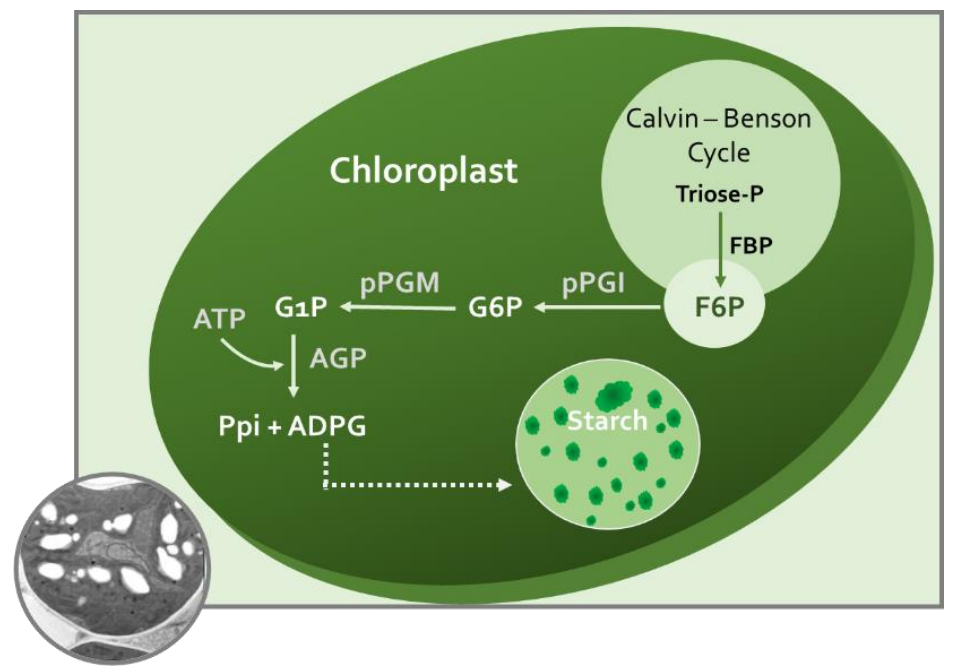

Figure 1. Graphic representation of the biosynthetic process for starch within a chloroplast. * Transmission electron micrographs of ultra-thin sections of flag leaves of rice plants taken from (Shen et al., 2019).

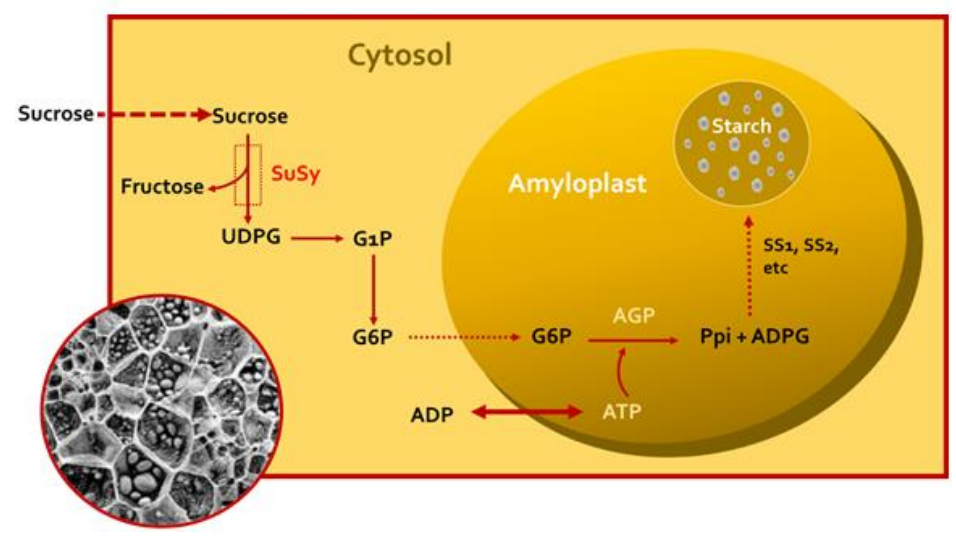

Figure 2. Graphic representation of the biosynthetic process for starch within an amyloplast. * Transmission electron micrographs of ultrathin sections of potato (Wise, 2007).

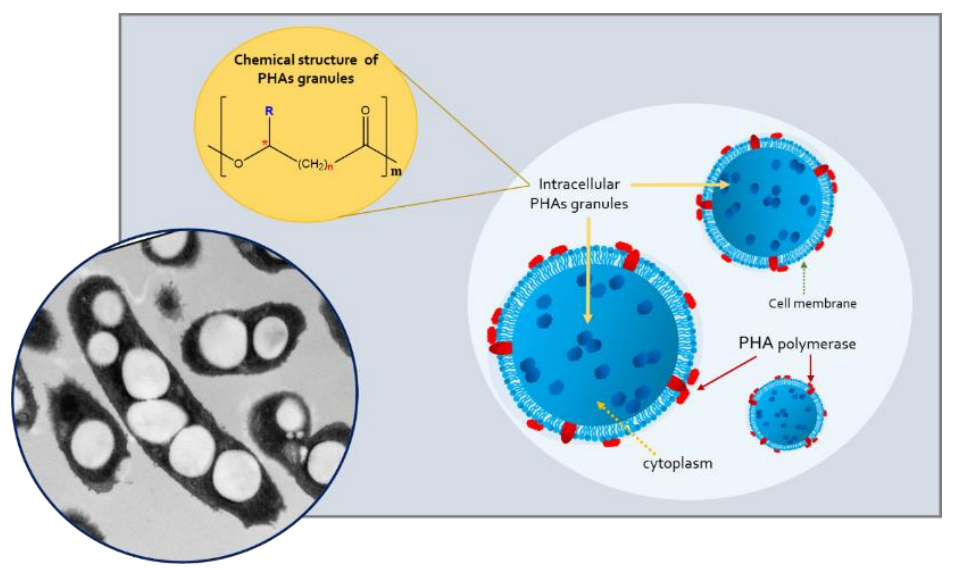

Figure 3. Diagram of the synthesis of PHAs in microorganisms. * TEM micrographs of C. necátor taken from (Sedlacek et al., 2019).
On the other hand, starch synthesis can be carried out within amyloplasts exhibiting some synthetic pathways alternate to those shown in the case of chloroplasts. One of the models considers the conversion of sucrose to starch where ADPG pyrophosphorylase (AGP) is the only source to obtain ADP-glucose (ADPG). Sucrose enters the cytosol and is degraded by sucrose synthase (SuSy) to produce fructose and UDP-glucose (UDPG), by means of UDPG pyrophosphorylase (UGP) UDPG is converted into G1P and PPi. Subsequently, G1P is metabolized to G6P by cytosolic phosphoglucomutase. Cytosolic G6P enters the amyloplasts, where it is converted to starch through the sequential activities of pPGM, AGP, and SS. It should be clarified that one of the differences that exist between chloroplasts and amyloplasts is that the latter cannot photosynthetically generate ATP, therefore, cytosolic ATP must enter the amyloplast to produce ADPG using AGP. The presence of this channel within the membranes of amyloplasts has been reported by several authors in different investigations. See Figure 2 (Bahaji et al., 2014; Subasinghe et al., 2014).

\section{Biosynthesis of poly(hydroxyalkanoates)}

PHAs are polymers based on hydroxyalkanoic acids synthesized inside the cell by microorganisms such as bacteria, archaea and algae for later use as a source of carbon and energy. The polymerization of hydroxyalkanoic acids results in biopolyesters by the action of intracellular enzymes. Generally, the reaction proceeds by condensation of the carboxyl group in the hydroxyalkanoic acid (monomer) with the hydroxyl group of another acid molecule, to form the ester bond. In most cases, microorganisms accumulate PHAs in the form of granules in the cytoplasm that are synthesized by the action of polymerase and depolymerase enzymes, see Figure 3 (Tarrahi et al., 2020).

The number of species capable of synthesizing is very diverse, and it is estimated that there are more than 300 species, microorganisms synthesize PHAs from substrates such as carbohydrates, aromatics, lipids, proteins, residues and gases. On the other hand, they also synthesize them if they are under stress situations such as limitations of nitrogen $(\mathrm{N})$, phosphorus $(\mathrm{P})$, sulfur $(\mathrm{S})$, magnesium $(\mathrm{Mg})$, oxygen (O) among others, see Table 1 (Chen et al., 2016; Yang et al., 2012).

Table 1. Substrates for several microorganisms

\begin{tabular}{ll}
\hline \multicolumn{1}{c}{ Bacteria } & \multicolumn{1}{c}{ Substrate } \\
\hline Rhodobacter sp. & Glucose \\
& Nitrogen and phosphorous stress \\
Thauera sp. & Sodium acetate \\
Alcaligenes sp. & Butyric and Valeric acids \\
& Glucose \\
& Milk waste \\
Pseudomonas sp. & Nitrogen stress \\
& Nonanoate \\
Azotobacter vinelandii & Decanoate \\
Azotobacter beijerinckii & Mg, N and P stress \\
\hline
\end{tabular}




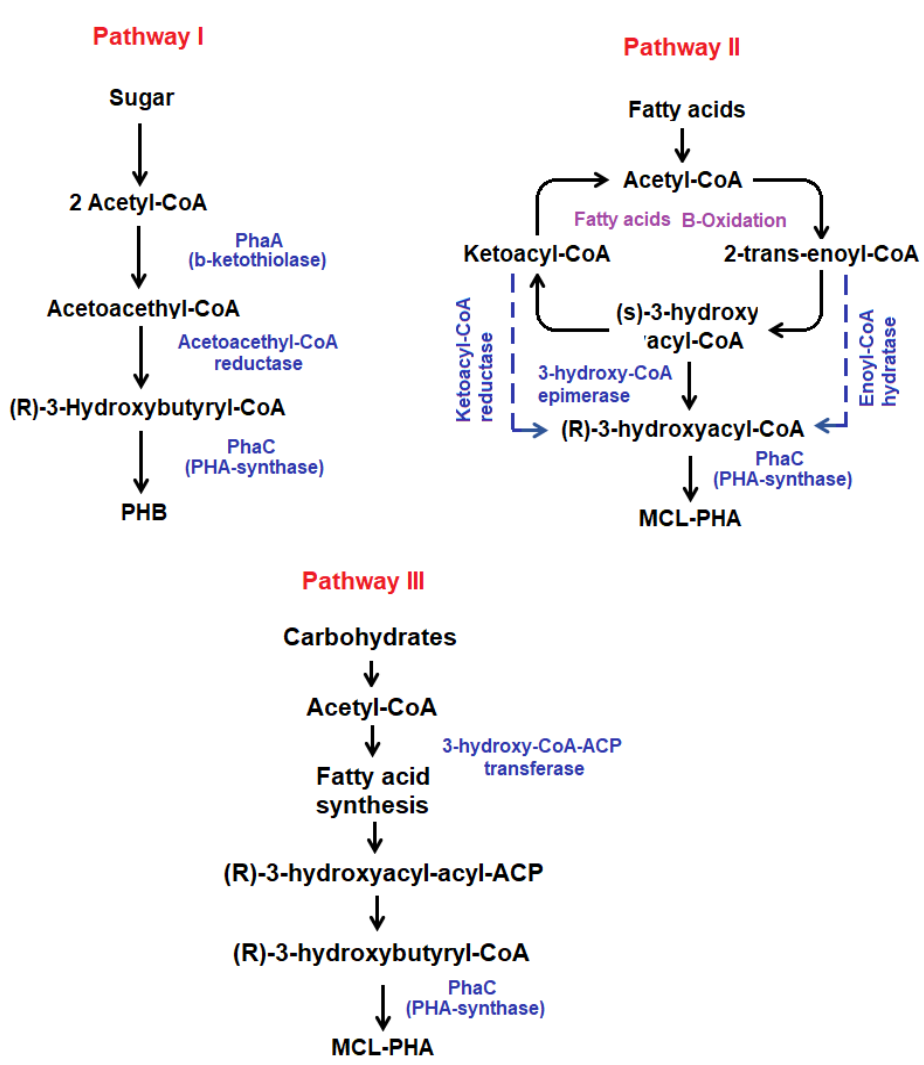

Figure 4. Metabolic pathways PHAs biosynthesis.

There are more than 14 metabolic pathways for the synthesis of PHAs, however there are three mostly reported. The first metabolic pathway begins from the sugar for the transformation into acetyl$\mathrm{CoA}$, then the condensation of two acetyl-CoA molecules and by the action of acetyl-coenzyme A acetyltransferases also known as $\beta$-ketothiolase leads to the synthesis of acetoacetyl-CoA which is subsequently reduced to 3-hydroxybutyryl-CoA by means of acetoacetyl-CoA reductase, finally PHA synthase (PhaC) is the key enzyme involved in the biosynthesis of PHAs and works by polymerizing monomeric hydroxyalkanoate substrates that in the first case derived from polyhydroxybutyrate (PHB). Path II begins from fatty acids that are transformed into acetyl-CoA and are used as a substrate to enter the $\beta$-oxidation cycle where 3 intermediate molecules are generated which are: (i) 2-trans-enoylCoA, (ii) (S) hydroxyacyl-CoA and (iii) ketoacyl-CoA and by action of 3ketoacyl-ACP reductase, 3-hydroxyacyl-CoA epimerase and enoylCoA hydratase respectively, the monomer of R-3-hydroxyacylCoA for synthesis of medium chain PHA (MCL-PHA) through (PhaC). Finally, the third route has as a substrate any type of carbohydrate that is transformed into acetyl-CoA, this molecule enters a route known as "novo of fatty acids" where the PhaG or (R) -3-hydroxyacyl-acyl-protein transporter-CoA transferase catalyzes the conversion of (R)-3-hydroxyacyl-ACP into (R)-3-hydroxyacylCoA so that finally MCL-PHA is obtained by the action of PhaC, see Figure 4 (Meng et al,2014; Agnew et al, 2013; Sudesh et al 2000).
PHAs are completely biodegradable and have similar properties to conventional fossil fuel plastics, making them suitable candidates for replacement of fossil fuel based plastics. These environmentally friendly bio-based bioplastics have great potential (Adeleye et al., 2020; Tarrahi et al., 2020). Currently, its large-scale production is still limited by its high cost of production, however, its high product cost must be countered by taking into account the environmental costs gained related to its use of traditional plastics. Among the most important factors in the total cost of PHA production, the use of pure or genetically modified crops, the cost of the raw materials used as precursors, and the recovery methods employed are the most important (Adeleye et al., 2020). Industrial processes for PHA production are based on the use of pure or genetically modified crops. Therefore, the costs of maintaining the culture, formulation of the substrate and sterilization of both the substrate and the reactor become key factors affecting the total cost of PHA production. As an alternative, the use of mixed microbial cultures (MMC) is promising because it will help reduce PHA production costs, since MMCs do not require sterile conditions and have a broader metabolic potential than individual strains (Mannina et al., 2019).

\section{Biosynthesis of poly(hydroxyalkanoates)}

In general, biocatalytic processes can be subdivided into: (I) fermentations that use natural carbon sources for the synthesis of new products, for example: such as alcohols, ketones, vitamins, antibiotics, or amino acids. These compounds are metabolites or final products because of a considerable number of chemical or enzymatic reactions by different microorganisms, (II) biotransformations that use a different strategy, where the precursor compound can be natural or unnatural and is converted into the new product, desired using the enzymatic potential of the microorganism through one (or a few) biochemical steps (Rizzarelli et al., 2020).

An example of biotransformation are the biodegradation processes which are defined as the decomposition of substances by the action of microorganisms. This action leads to the recycling of carbon, the mineralization of organic compounds for the generation of new biomass. The biodegradation process includes several steps: (i) Biodeterioration, which corresponds to the combined action of microbial communities, other decomposing organisms and / or abiotic factors that achieve the fragmentation of the starting material; (ii) Depolymerization, occurs with the secretion of catalytic agents such as enzymes and free radicals that progressively reduce the molecular weight of the polymeric fragments and generate oligomers, dimers and monomers; (iii) Assimilation, the products obtained as a result of the previous fragmentation are incorporated into the microbial metabolism to produce primary and secondary metabolites, energy and new biomass; (iv) Mineralization, some simple or complex metabolites can be excreted to the extracellular environment (for example, organic acids, aldehydes, terpenes, antibiotics, etc.), which are released into the environment simple molecules such as $\mathrm{CO}_{2}, \mathrm{~N}_{2}, \mathrm{CH}_{4}, \mathrm{H}_{2} \mathrm{O}$ and different salts of fully oxidized intracellular metabolites (Rizzarelli et al, 2020; Konkol et al, 2019). 
Finally, another example of biotransformation are biotechnological processes for the partial degradation or transformation of rubber (poly(1,4-isoprene)), gram-positive species of Streptomyces that degrade rubber, such as $S$. coelicolor $1 A, S$. griseus $1 D$ and Streptomyces sp. K30 enzyme "latex cleaning protein" (Lcp) to transform the polymer into more hydrophilic oligoisoprenoids. In addition, gram-negative species such as $S$. cummioxidans $35 Y$ and R. gummiphilus NS21, other rubber-degrading microorganisms such as Gordonia polyisoprenivorans VH2, Gordonia westfalica Kb2, Nocardia farcinica (Andler et al., 2020).

\section{Conclusions}

Starch and PHAs are promissory biopolymers for making of bioplastics and other important applications. Their biosynthetic pathways are complex and well-known, therefore, studies aimed to the control and improving of biosynthetic processes and biotransformations associated with them are possible.

In particular, starch can be easily extracted from several types of plants whereas PHAs can be biotechnologically obtained.

:+:

Conflict interest. Authors declare that there is no conflict of interest regarding the publication of this paper.

Acknowledgements. V. Garcés and M. Palencia thanks to University del Valle and Mindtech s.a.s. (AFICAT MT-UV 2018-2023).

\section{References}

Adeleye, A. T., et al. Sustainable synthesis and applications of polyhydroxyalkanoates (PHAs) from biomass. Process Biochemistry. 96 (2020), 174-193. https://doi.org/10.1016/j.procbio.2020.05.032

Agnew, D. E., Pfleger, B. F. Synthetic biology strategies for synthesizing polyhydroxyalkanoates from unrelated carbon sources. Chemical Engineering Science, 103 (2013), 58-67. https://doi.org/10.1016/j.ces.2012.12.023

Andler, R., et al. Biotransformation of poly (cis-1, 4-isoprene) in a multiphase enzymatic reactor for continuous extraction of oligoisoprenoid molecules. New Biotechnology, 58 (2020), 10-16.

Bahaji, A., et al. Starch biosynthesis, its regulation and biotechnological approaches to improve crop yields. Biotechnology Advances, 32(1) (2014), 87-106. https://doi.org/10.1016/j.biotechadv.2013.06.006

Chen, G. Q., et al. Synthetic biology of microbes synthesizing polyhydroxyalkanoates (PHA). Synthetic and Systems Biotechnology, 1(4) (2016), 236-242. https://doi.org/10.1016/j.synbio.2016.09.006

Chen, Y. F., et al. Chemical modification of starch. In Starch in food. Woodhead Publishing, (2018), 283-321. https://doi.org/10.1016/B9780-08-100868-3.00007-X

Clasen, S. H., et al. Synthesis and characterization of cassava starch with maleic acid derivatives by etherification reaction. Carbohydrate polymers, 180 (2018), 348-353.

Haroon, M., et al. Chemical modification of starch and its application as an adsorbent material. Rsc Advances, 6(82) (2016), 78264-78285. https://doi.org/10.1039/C6RA16795K

IUPAC. Compendium of Chemical Terminology, 2nd ed. (the "Gold Book"). Compiled by A. D. McNaught and A. Wilkinson. Blackwell Scientific Publications, Oxford (1997). Online version (2019-) created by S. J. Chalk. ISBN 0-9678550-9-8. https://doi.org/10.1351/goldbook

Jendrossek, D., Birke, JRubber oxygenases. Applied microbiology and biotechnology, 103 (2019), 125-142.

Karan, H., et al. Green bioplastics as part of a circular bioeconomy. Trends in plant science, 24(3) (2019), $237-249$. https://doi.org/10.1016/j.tplants.2018.11.010

Konkol, D., et al. Biotransformation of rapeseed meal leading to production of polymers, biosurfactants, and fodder. Bioorganic chemistry, 93 (2019), 102865. https://doi.org/10.1016/j.bioorg.2019.03.039 
Kosseva, M. R., et al. Biopolymers produced from food wastes: a case study on biosynthesis of bacterial cellulose from fruit juices. In Food Industry Wastes. Academic Press, (2020), 225-254. https://doi.org/10.1016/B978-0-12-817121-9.00011-5

Li, H., et al. Starch branching enzymes contributing to amylose and amylopectin fine structure in wheat. Carbohydrate polymers, 224 (2019), 115185. https://doi.org/10.1016/j.carbpol.2019.115185

Mannina, G., et al. Bioplastic recovery from wastewater: a new protocol for polyhydroxyalkanoates (PHA) extraction from mixed microbial cultures. Bioresource technology, 282 (2019), 361-369. https://doi.org/10.1016/j.biortech.2019.03.037

Meng, D. C., et al. Engineering the diversity of polyesters. Current opinion in biotechnology, 29 (2014), 24-33. https://doi.org/10.1016/j.copbio.2014.02.013

Miao, H., et al. Identification of genes encoding granule-bound starch synthase involved in amylose metabolism in banana fruit. PloS one, 9(2) (2014), e88077. https://doi.org/10.1371/journal.pone.0088077

Nakayama, A., et al. Biodegradation in seawater of aliphatic polyesters. Polymer Degradation and Stability, 166 (2019), $290-299$. https://doi.org/10.1016/j.polymdegradstab.2019.06.006

Orzechowski, S. Starch metabolism in leaves. Acta biochimica polonica, 55(3) (2008)., 435-445.

Rizzarelli, P., et al. Biodegradation of green polymer composites: Laboratory procedures and standard test methods. In Advanced Applications of Bio-Degradable Green Composites. Materials Research Forum LLC, (2020), 1-44.

Sedlacek, P., et al. PHA granules help bacterial cells to preserve cell integrity when exposed to sudden osmotic imbalances. New biotechnology, 49 (2019), 129-136. https://doi.org/10.1016/j.nbt.2018.10.005

Shen, B. R., et al. Engineering a new chloroplastic photorespiratory bypass to increase photosynthetic efficiency and productivity in rice. Molecular plant, 12(2) (2019), 199-214. https://doi.org/10.1016/j.molp.2018.11.013

Subasinghe, R. M., et al. Multimeric states of starch phosphorylase determine protein-protein interactions with starch biosynthetic enzymes in amyloplasts. Plant Physiology and Biochemistry, (2014) 83, 168-179. https://doi.org/10.1016/j.plaphy.2014.07.016

Sudesh, K., et al. Synthesis, structure and properties of polyhydroxyalkanoates: biological polyesters. Progress in polymer science, 25(10) (2000), 1503-1555. https://doi.org/10.1016/S0079-6700(00)00035-6

Tarrahi, R., et al. Polyhydroxyalkanoates (PHA): from production to nanoarchitecture. International Journal of Biological Macromolecules, 146 (2020), 596-619. https://doi.org/10.1016/j.ijbiomac.2019.12.181

Tetlow, I. J., Emes, M. J. Starch biosynthesis in higher plants: the enzymes of starch synthesis. 4(2011), 58-76. https://doi.org/10.1016/B978$\underline{0-444-64046-8.00213-5}$

Varela, G., Grotiuz, G. Fisiología y metabolismo bacteriano. Uruguay, Editorial Cefa, (2008), 43-58.

Verma, M. L., et al. Microbial production of biopolymers with potential biotechnological applications. In Biopolymer-Based Formulations. Elsevier, (2020), 105-137. https://doi.org/10.1016/B978-0-12-816897-4.00005-9

Wang, S., et al. Starch Modification and Application. In Starch Structure, Functionality and Application in Foods, (2020), 131-149.

Wise, R. R. The diversity of plastid form and function. In The structure and function of plastids. Springer, Dordrecht, (2007), 3-26.

Yang, X., et al. Enriching polyhydroxyalkanoates (PHA) producing microorganisms by complex organics from quickly alkaline fermentation liquor. Journal of the Taiwan Institute of Chemical Engineers, 43(6) (2012)., 953-957. https://doi.org/10.1016/j.jtice.2012.06.004

:+:

(C) MT-Pallantia Publisher (2020) 